TRANSACTIONS OF THE

AMERICAN MATHEMATICAL SOCIETY

Volume 284. Number 2. August 1984

\title{
INFINITE CROSSED PRODUCTS AND GROUP-GRADED RINGS
}

BY

\author{
D. S. PASSMAN ${ }^{1}$
}

\begin{abstract}
In this paper, we precisely determine when a crossed product $R * G$ is semiprime or prime. Indeed we show that these conditions ultimately depend upon the analogous conditions for the crossed products $R * N$ of the finite subgroups $N$ of $G$ and upon the interrelationship between the normalizers of these subgroups and the ideal structure of $R$. The proof offered here is combinatorial in nature, using the $\Delta$-methods, and is entirely self-contained. Furthermore, since the argument applies equally well to strongly $G$-graded rings, we have opted to work in this more general context.
\end{abstract}

Let $G$ be a multiplicative group and let $R$ be a ring with 1 . Then a crossed product $R * G$ of $G$ over $R$ is an associative ring determined by $G, R$ and certain other parameters. To be more precise, for each $x \in G$ there exists an element $\bar{x} \in R * G$ and every element $\alpha \in R * G$ is uniquely writable as a finite sum

$$
\alpha=\sum_{x \in G} r_{x} \bar{x}
$$

with $r_{x} \in R$. Addition in $R * G$ is componentwise and multiplication is given by the formulas

$$
\bar{x} \bar{y}=t(x, y) \overline{x y}, \quad r \bar{x}=\bar{x} r^{\bar{x}}
$$

for all $x, y \in G$ and $r \in R$. Here $t: G \times G \rightarrow U$ is a map from $G \times G$ to the group of units $U$ of $R$ and, for fixed $x \in G$, the map ${ }^{\bar{x}}: r \rightarrow r^{\bar{x}}$ is an automorphism of $R$.

It is a simple exercise to determine the relations on $t$ and the automorphisms ${ }^{\bar{x}}$ which make $R * G$ associative. From this it follows easily that $R * G$ has an identity element, namely $1=t(1,1)^{-1} \overline{1}$, and that each $\bar{x}$ is invertible. Indeed

$$
\mathfrak{S}=\{u \bar{x} \mid u \in U, x \in G\}
$$

is a multiplicative group of units in $R * G$, the group of trivial units. Thus the equation $r \bar{x}=\bar{x} r^{\bar{x}}$ is equivalent to $\bar{x}^{-1} r \bar{x}=r^{\bar{x}}$ and hence the automorphism ${ }^{\bar{x}}$ is merely conjugation by a unit in $R * G$. In fact, it is clear that $\mathbb{S}$ acts on $R$ by conjugation. In general, $R * G$ does not contain an isomorphic copy of $G$. However, we do have $R \subseteq R * G$ by way of the embedding $r \rightarrow r 1$ and then $U \triangleleft$ (S) and (5) $U \simeq G$.

Received by the editors May 31, 1983 and, in revised form, December 6, 1983.

1980 Mathematics Subject Classification. Primary 16A27, 16A03.

${ }^{1}$ Research supported in part by NSF Grant No. MCS 80-02773. 
An interesting problem, studied for the past 20 years, concerns finding reasonable necessary and sufficient conditions for $R * G$ to be semiprime or prime. In the case of ordinary group algebras these are results of the author [13] and of I. G. Connell [4], respectively. They were obtained using a coset counting argument known as the $\Delta$-method, which effectively reduced the question to the finite normal subgroups of $G$. Furthermore, this same technique handled twisted group algebras with little additional difficulty [14]. However, when $G$ acts nontrivially on the ring $R$, another dimension is added to the problem. Here the first result, due to G. Azumaya [1] showed that if $R$ is a simple ring and $G$ is outer on $R$, then $R * G$ is simple. S. Montgomery [9] proved that if $R$ is prime (or semiprime) and $G$ is $X$-outer on $R$, then $R * G$ is prime (or semiprime, respectively). In [6], J. Fisher and S. Montgomery settled the semiprime question for $G$ finite. Infinite groups were considered by $\mathrm{S}$. Montgomery and the author in [10] where the $\Delta$-methods and the techniques of [6] combined to handle the case where $R$ is a prime ring. This was extended in [16] to semiprime coefficient rings and then the problem was essentially solved in [18].

It was apparent from the work of [18] that the semiprime and prime condition for $R * G$ ultimately depends on the analogous condition for certain crossed products of finite subgroups of $G$. In this paper, we give a precise formulation of this fact and the main result is

Theorem. Let $R * G$ be a crossed product of $G$ over $R$. Then $R * G$ contains nonzero ideals $A, B$ with $A B=0$ if and only if there exist:

(i) subgroups $N \triangleleft H \subseteq G$ with $N$ finite,

(ii) an $H$-invariant ideal $I$ of $R$ with $I^{x} I=0$ for all $x \in G \backslash H$,

(iii) nonzero $H$-invariant ideals $\tilde{A}, \tilde{B}$ of $R * N$ with $\tilde{A}, \tilde{B} \subseteq I * N$ and $\tilde{A} \tilde{B}=0$.

Furthermore, $A=B$ if and only if $\tilde{A}=\tilde{B}$.

The results of [18] are immediate corollaries of the above.

The proof of the main theorem is entirely combinatorial in nature, using the $\Delta$-methods and a modification of the bookkeeping procedure of [18]. Indeed the proof is quite similar to the work of the latter paper. Nevertheless, crossed products with operators and the ideals $\mathscr{S}(H)$ and $\mathscr{P}(H)$ which played such a prominent role in the earlier argument no longer occur. Moreover, the results of [10] and [16] are no longer needed. Because of these many simplifications and because of the delicate nature of the proof, we have opted to offer a completely self-contained version here. We hope this will make the paper more readable and the proof more understandable.

For a number of reasons, we have decided to work in the more general context of strongly $G$-graded rings. First, there is at present a good deal of interest in these rings and in their prime and nilpotent ideals $[2,3,5,11,12]$. Second, the proof is no harder in this generality and in fact certain aspects, for example the group action on the ideals of $R$, actually become more natural. Third, it forced us to rethink and simplify a number of arguments which seemed to depend upon the existence of the units $\bar{x}$. Finally, it allowed us to further separate this paper from its predecessor [18]. 
1. Group-graded rings. Let $G$ be a multiplicative group. An associative ring $S$ is said to be $G$-graded if $S$ can be written as $S=\oplus \sum_{x \in G} R_{x}$, the direct sum of additive subgroups $R_{x}$, indexed by the elements of $G$, with $R_{x} R_{y} \subseteq R_{x y}$ for all $x, y \in G$. It is clear that $R_{1}=R$ is a subring of $S$ and we assume throughout that $1 \in R$ and that the unit element of $R$ is also the unit element of $S$. It therefore follows that each $R_{x}$ is a unital $(R, R)$-bimodule.

If $\alpha \in S$, we can write $\alpha$ as the finite sum $\alpha=\sum \alpha_{x}$ with $\alpha_{x} \in R_{x}$. The summands $\alpha_{x}$ are then the homogeneous components of $\alpha$ and the support of $\alpha$ is $\operatorname{Supp} \alpha=\{x$ $\left.\in G \mid \alpha_{x} \neq 0\right\}$. In general, we will subscript homogeneous elements of $S$ to indicate their grade.

The $G$-graded ring $S$ is said to be strongly $G$-graded if $R_{x} R_{y}=R_{x y}$ for all $x, y \in G$. As is well known, this condition follows if we merely assume that $R_{x} R_{x^{-1}}=R_{1}$ for all $x$. Furthermore, it is clear that any crossed product over $G$ is a strongly $G$-graded ring in a natural way. By a terrible abuse of notation, we will also denote strongly $G$-graded rings by $R * G$. Thus

$$
R * G=\bigoplus \sum_{x \in G} R_{x}
$$

with $R_{1}=R$. In other words, the notation merely indicates the group $G$ and the base ring $R=R_{1}$; all the remaining structural information is somehow embedded in the star. As with crossed products, we use this with the understanding that if $H$ is a subgroup of $G$, then $R * H$ is the natural strongly $H$-graded subring given by

$$
R * H=\bigoplus \sum_{x \in H} R_{x} .
$$

In this section, we make a few elementary observations, state the main theorem for group-graded rings and then prove that result in the trivial direction. We assume throughout that $R * G$ is a fixed strongly $G$-graded ring.

LEMMA 1.1. With the above notation:

(i) The right and left annihilators of any $R_{x}$ are zero.

(ii) if $\alpha_{y}$ is a nonzero element of $R_{y}$ and $x y z=1$, then $R_{x} \alpha_{y} R_{z}$ is a nonzero ideal of $R$.

PRoof. (i) is clear since $1 \in R_{1}=R_{x} R_{x^{-1}}=R_{x^{-1}} R_{x}$ and (ii) is immediate since $R_{x} \alpha_{y} R_{z}$ is a nonzero $(R, R)$-subbimodule of $R=R_{1}$.

We will use this lemma, and its obvious generalizations, freely throughout the remainder of this paper.

Now suppose $N \triangleleft H \subseteq G$. For $x \in H$ and $I$ an ideal of $R * N$, we define $I^{x}=$ $R_{x^{-1}} I R_{x}$. In this way, $H$ acts on the ideals of $R * N$ and basic properties are as follows.

LEMMA 1.2. Let $N \triangleright H \subseteq G$, let $x, y \in H$ and let $I, J$ be ideals of $R * N$. Then:

(i) $I^{x}$ is an ideal of $R * N$;

(ii) $\left(I^{x}\right)^{y}=1^{x y}, I^{1}=I$;

(iii) if $I \subseteq J$, then $I^{x} \subseteq J^{x}$;

(iv) $(I J)^{x}=I^{x} J^{x}$. 
Proof. Since $N \triangleright H$ and $x \in H$, it follows easily that $(R * N) R_{x}=R_{x}(R * N)$. From this we conclude first that $I^{x} \subseteq R * N$ and then that $I^{x}$ is an ideal of this subring. Now for (ii) and (iv) we have

$$
\left(I^{x}\right)^{y}=R_{y^{-1}} I^{x} R_{y}=R_{y^{-1}}\left(R_{x^{-1}} I R_{x}\right) R_{y}=R_{(x y)^{-1}} I R_{x y}=I^{x y}
$$

and

$$
I^{x} J^{x}=\left(R_{x^{-1}} I R_{x}\right)\left(R_{x^{-1}} J R_{x}\right)=R_{x^{-1}}(I R J) R_{x}=(I J)^{x}
$$

since $I R=I$. Finally, (iii) is obvious so the lemma is proved.

If we let $\mathscr{I}$ denote the set of ideals of $R * N$ in the above situation, then (i) and (ii) assert that the map $x \rightarrow^{x}$ is a homomorphism of $G$ into $\operatorname{Sym}(\mathscr{I})$. Furthermore, by (iii) these are inclusion preserving permutations and in particular they preserve the lattice operations of arbitrary intersections and sums. (iv) says that finite products are also preserved.

Continuing with this notation, an ideal $I$ of $R * N$ is said to be $H$-invariant if $I^{x}=I$ for all $x \in H$. Since $R_{x} R_{x^{-1}}=R_{x^{-1}} R_{x}=R$, this clearly occurs if and only if $I R_{x}=R_{x} I$ for all $x \in H$.

Note that if $N=\langle 1\rangle$, then $R * N=R$ and, in this way, $G$ acts on the ideals of $R$. With this understanding we can now state the main result for group-graded rings.

THEOREM 1.3. Let $R * G$ be a strongly $G$-graded ring with base ring $R$. Then $R * G$ contains nonzero ideals $A, B$ with $A B=0$ if and only if there exist:

(i) subgroups $N \triangleleft H \subseteq G$ with $N$ finite,

(ii) an $H$-invariant ideal $I$ of $R$ with $I^{x} I=0$ for all $x \in G \backslash H$,

(iii) nonzero $H$-invariant ideals $\tilde{A}, \tilde{B}$ of $R * N$ with $\tilde{A}, \tilde{B} \subseteq I(R * N)$ and $\tilde{A} \tilde{B}=0$.

Furthermore $A=B$ if and only if $\tilde{A}=\tilde{B}$.

Note that if $R * G$ is a crossed product, then the permutation action on the ideals as defined above is merely conjugation by the group of trivial units. Thus Theorem 1.3 does generalize the result stated in the introduction. It is convenient to record the following elementary

Lemma 1.4. Let $H$ be a subgroup of $G$ and suppose that $I$ is an ideal of $R$ with $I^{x} I=0$ for all $x \in G \backslash H$. Then:

(i) $I R_{x} I=0$ for all $x \in G \backslash H$,

(ii) $I(R * G) I \subseteq I(R * H) \subseteq R * H$. have

Proof. (i) is clear since $\underline{r}\left(R_{x^{-1}}\right)=0$. Moreover, since $R * G=\oplus \sum_{x \in G} R_{x}$, we

$$
I(R * G) I=\bigoplus \sum_{x \in G} I R_{x} I=\bigoplus \sum_{x \in H} I R_{x} I \subseteq I(R * H) \subseteq R * H
$$

and (ii) is proved.

We can now offer the

Proof of Theorem 1.3 (EASY Direction). Here we assume that $R * G$ is given and that $H, N, I, \tilde{A}$ and $\tilde{B}$ exist and satisfy the appropriate properties. We set $A=$ $(R * G) \tilde{A}(R * G)$ and $B=(R * G) \tilde{B}(R * G)$ so that these are nonzero ideals of the 
strongly $G$-graded ring $R * G$. The goal is to show that $A B=0$ or equivalently that $\tilde{A} R_{x} \tilde{B}=0$ for all $x \in G$.

If $x \in H$, then since $\tilde{A}$ is $H$-invariant, we have

$$
\tilde{A} R_{x} \tilde{B}=R_{x} \tilde{A} \tilde{B}=0 .
$$

On the other hand, if $x \in G \backslash H$, then since $\tilde{A}, \tilde{B} \subseteq I(R * N)$ we have

$$
\tilde{A} R_{x} \tilde{B} \subseteq I(R * N) R_{x} I \cdot(R * N) .
$$

But $(R * N) R_{x}=\sum_{y \in N x} R_{y}$ and $N x \subseteq G \backslash H$ so Lemma 1.4(i) yields $I(R * N) R_{x} I$ $=0$. Therefore $\tilde{A} R_{x} \tilde{B}=0$ and we have shown that $A B=0$. Since $\tilde{A}=\tilde{B}$ implies $A=B$, this direction is proved.

Most of the remainder of this paper is devoted to proving the opposite direction.

2. Coset calculus. Let $H$ be a group and let $R$ be a ring. We say that $H$ strongly permutes the ideals of $R$ if for all $x, y \in H$ and ideals $I, J$ of $R$ we have:

(i) $I^{x}$ is an ideal of $R$;

(ii) $\left(I^{x}\right)^{y}=I^{x y}, I^{1}=I$;

(iii) if $I \subseteq J$ then $I^{x} \subseteq J^{x}$;

(iv) $(I J)^{x}=I^{x} J^{x}$.

Thus, for example, if we are given the strongly $H$-graded ring $R * H$, then Lemma 1.2 asserts precisely that $H$ strongly permutes the ideals of $R$. As we observed previously, the first three conditions above imply that the action comes from a homomorphism of $H$ into the group of inclusion preserving permutations on the set of ideals of $R$. In particular, they also preserve the lattice operations of arbitrary sums and intersections. Thus we also have $0^{x}=0$ and $R^{x}=R$ for all $x \in H$.

Now let $J$ be a nonzero ideal of $R$. In view of the conclusion of Theorem 1.3, we are obviously interested in studying those $h \in H$ with $J^{h} J=0$. Now the $\Delta$-methods usually give information which asserts that something occurs for all elements of $H$ except for those in a finite union of cosets of varying subgroups. In this section we show how to reformulate such a conclusion so that it in fact holds for all elements in $H \backslash L$ where $L$ is a single subgroup of $H$ suitably determined by the situation.

Suppose $A$ and $B$ are subgroups of $H$ and that the left cosets $x A$ and $y B$ are not disjoint. If $z \in x A \cap y B$, then $x A=z A, y B=z B$ and hence

$$
x A \cap y B=z A \cap z B=z(A \cap B) .
$$

In other words, the intersection of two left cosets is either empty or a coset of the intersection. Property (iv) above will be crucial in the next two lemmas.

LEMMA 2.1. Let $H$ strongly permute the ideals of $R$ and let $J$ be a nonzero ideal of $R$ such that

$$
J^{h} J=0 \quad \text { for all } h \in H \backslash \bigcup_{1}^{n} h_{k} H_{k} .
$$

Here $\bigcup_{1}^{n} h_{k} H_{k}$ is a fixed finite union of left cosets of the subgroups $H_{k}$ of $H$. Then there exists a subgroup $L$ of $H$ and a nonzero product $0 \neq K=J^{v_{1}} J^{y_{2}} \ldots J^{y_{r}}$ of $H$-conjugates of $J$, with some $y_{i}=1$, such that $K^{h} K=0$ for all $h \in H \backslash L$. Furthermore $\left|L: L \cap H_{k}\right|<\infty$ for some $k$. 
Proof. In the course of this proof we will replace $\left\{H_{1}, H_{2}, \ldots, H_{n}\right\}$ by certain other finite sets $\mathfrak{A}$ of subgroups of $H$ with the property that $A \in \mathfrak{A}$ implies $A \subseteq H_{k}$ for some $k$. We note that if the result is proved for such a set $\mathfrak{A}$, then from $|L: L \cap A|<\infty$ for some $A$ and $L \cap A \subseteq L \cap H_{k}$ we obtain $\left|L: L \cap H_{k}\right|<\infty$. In other words, the result will then follow for the original subgroups $H_{1}, H_{2}, \ldots, H_{n}$.

If $\mathfrak{A}$ is the set of all proper (that is, nonempty) intersections of the $H_{k}$ 's, then $\mathfrak{A}$ is finite and closed under intersections. Thus without loss of generality we can now assume that the $H_{k}$ 's are contained in a finite set $\mathfrak{A}$ closed under intersections and we prove the result by induction on $|\mathfrak{A}| \geqslant 0$. If $|\mathfrak{A}|=0$ then the hypothesis and conclusion both assert that $J^{h} J=0$ for all $h \in H$.

Assume now that $|\mathfrak{A}| \geqslant 1$, let $A$ be a maximal member of $\mathfrak{A}$ and set $\mathfrak{U}^{\prime}=\mathfrak{A} \backslash\{A\}$. Then $\left|\mathfrak{A}{ }^{\prime}\right|<|\mathfrak{A}|$ and $\mathfrak{A}^{\prime}$ is closed under intersections. We will be concerned with finite unions of left cosets $S=\bigcup h_{i j} A_{i}$ with $A_{i} \in \mathfrak{A}$. By the support of $S$ we mean those $A_{i}$ 's which occur in this representation. Suppose $K=J^{y_{1}} J^{y_{2}} \cdots J^{y_{r}} \neq 0$, some $y_{i}=1$, and $K^{h} K=0$ for all $h \in H \backslash S$. If $A \notin \operatorname{Supp} S$, then $\operatorname{Supp} S \subseteq \mathfrak{U}^{\prime}$ and induction applies. Thus there exists a finite product $I=K^{u_{1}} K^{u_{2}} \cdots K^{u_{s}} \neq 0$ with some $u_{j}=1$ and $I^{h} I=0$ for all $h \in H \backslash L$. Since $I$ is also a suitable product of conjugates of $J$ and since $\left|L: L \cap A_{t}\right|<\infty$ for some $A_{t} \in \mathfrak{U}^{\prime}$, the result follows in this case.

Thus we can assume, for all such pairs $K$ and $S$ as above, that $A \in \operatorname{Supp} S$. Of course there is at least one such pair by hypothesis and now we choose $K$ and $S$ so that $S$ has the smallest number, say $m \geqslant 1$, of cosets of $A$ occurring in its representation. Then

$$
S=z_{1} A \cup z_{2} A \cup \cdots \cup z_{m} A \cup T
$$

where $T$ is a finite union of cosets of groups in $\mathfrak{U}^{\prime}$ and we define $L$ by

$$
L=\left\{h \in H \mid h\left(\bigcup_{1}^{m} z_{i} A\right)=\bigcup_{1}^{m} z_{i} A\right\} .
$$

Suppose $K^{x} K \neq 0$ for some $x \in H$. Then $K^{x} K$ is a nonzero finite product of $H$-conjugates of $J$ with some conjugating element equal to 1 . Furthermore the symbolic formula $K^{(H \backslash S)} K=0$ yields $K^{x \cdot x^{-1}(H \backslash S)} K=0$ so $\left(K^{x} K\right)^{h}\left(K^{x} K\right)=0$ for all $h$ with

$$
h \in(H \backslash S) \cup x^{-1}(H \backslash S)=H \backslash\left(S \cap x^{-1} S\right) .
$$

Since $\mathfrak{A}$ is closed under intersections, it is clear that $S \cap x^{-1} S$ is also a finite union of left cosets of members of $\mathfrak{A}$. Indeed, since clearly $\operatorname{Supp} x^{-1} S=\operatorname{Supp} S, S \cap x^{-1} S$ is a union of cosets of groups of the form $B \cap C$ with $B, C \in \operatorname{Supp} S$. Furthermore, since $A$ is a maximal member of $\mathfrak{A}$, we note that $A=B \cap C$ can occur if and only if $B=C=A$. By definition of $m, S \cap x^{-1} S$ contains $m^{\prime} \geqslant m$ cosets of $A$.

Since

$$
x^{-1} S=x^{-1} z_{1} A \cup x^{-1} z_{2} A \cup \cdots \cup x^{-1} z_{m} A \cup x^{-1} T
$$


the $A$-cosets of $S \cap x^{-1} S$ come from

$$
\left(\bigcup_{1}^{m} z_{i} A\right) \cap\left(\bigcup_{1}^{m} x^{-1} z_{i} A\right) .
$$

But cosets of $A$ are either disjoint or identical so this intersection has $m^{\prime} \leqslant m$ terms. Thus we must have $m^{\prime}=m$ so

$$
\bigcup_{1}^{m} z_{i} A=x^{-1}\left(\bigcup_{1}^{m} z_{i} A\right)
$$

and hence $x \in L$. In other words, we have shown that $K^{(H \backslash L)} K=0$

Now $H$ permutes the set $\Omega$ of left cosets of $A$ by left multiplication and $L$ is the set stabilizer of the finite subset $\Lambda=\left\{z_{1} A, z_{2} A, \ldots, z_{m} A\right\}$ of $\Omega$. Thus $L$ is a subgroup of $H$. Moreover if $\alpha=z A \in \Lambda$, then $H_{\alpha}=\{h \in H \mid h \alpha=\alpha\}=z A z^{-1}$ and it follows that $\left|L: L \cap\left(z A z^{-1}\right)\right|<\infty$. We can eliminate this conjugating element $z^{-1}$ by conjugating both $L$ and $K$ by $z$. However in so doing we lose the property of $K$ that some $y_{i}=1$. Thus we must take a different approach.

Suppose first that $L \cap z_{i} A=\varnothing$ for all $i=1,2, \ldots, m$. Then $K^{h} K=0$ for all $h$ with

$$
h \in(H \backslash S) \cup(H \backslash L)=H \backslash(L \cap S)
$$

and, by the above assumption, $L \cap S=L \cap T$ is a finite union of cosets of the groups in $\mathfrak{B}=\left\{L \cap \tilde{A} \mid \tilde{A} \in \mathfrak{U}^{\prime}\right\}$. Since $|\mathfrak{P}| \leqslant\left|\mathfrak{H}^{\prime}\right|<|\mathfrak{A}|$ and since $\mathfrak{P}$ is clearly closed under intersections, induction applies here. Thus, as before, there exists $I=K^{u_{1}} K^{u_{2}}$ $\cdots K^{u_{s}} \neq 0$ with some $u_{j}=1$ such that $I^{h} I=0$ for all $h \in H \backslash \tilde{L}$ with $|\tilde{L}: \tilde{L} \cap B|$ $<\infty$ for some $B \in \mathfrak{B}$. Since $I$ has the appropriate form, the result follows in this case.

Finally, if $L \cap z_{i} A \neq \varnothing$ for some $i$, we may assume that $z=z_{i} \in L$. Since $\left|L: L \cap\left(z A z^{-1}\right)\right|<\infty$, conjugating this expression by $z \in L$ then yields $|L: L \cap A|$ $<\infty$ and the lemma is proved.

We remark that the same result holds with $J^{h} J$ replaced by $J J^{h}$. Indeed, in the proof, merely replace all occurrences of $K^{h} K$ by $K K^{h}$. Furthermore both of these hold if left cosets are replaced by right cosets. Merely note that $J^{h} J=0$ is equivalent to $J J^{h^{-1}}=0$ and that replacing $h$ by $h^{-1}$ effectively interchanges right and left cosets. The form of the lemma proved here is the one we will use.

Moreover, suppose that $H=\bigcup_{1}^{n} h_{k} H_{k}$ is given, let $H$ act trivially on the ideals of any ring $R$ with 1 and take $J=R$. Then the hypothesis of the above lemma is vacuously satisfied and the conclusion implies that $R^{h} R=0$ for all $h \in H \backslash L$. Thus we must have $L=H$ and $\left|H: H_{k}\right|<\infty$ for some $k$. In other words, Lemma 2.1 generalizes the well-known result concerning the existence of subgroups of finite index. We actually require the following slight strengthening of the above.

LEMMA 2.2. Let $H$ strongly permute the ideals of $R$ and let $J$ be a nonzero ideal of $R$ such that

$$
J^{h} J=0 \quad \text { for all } h \in W \backslash \bigcup_{1}^{n} w_{k} H_{k}
$$


Here $W$ is a subgroup of $H$ of finite index and $\bigcup_{1}^{n} w_{k} H_{k}$ is a fixed finite union of left cosets of the subgroups $H_{k}$ of $W$. Then there exists a subgroup $L$ of $H$ and a nonzero

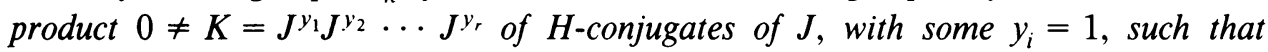
$K^{h} K=0$ for all $h \in H \backslash L$. Furthermore, $\left|L: L \cap H_{k}\right|<\infty$ for some $k$.

Proof. Since $|H: W|<\infty$ and $J \neq 0$ we can choose the sequence $\Lambda=$ $\left\{h_{1}, h_{2}, \ldots, h_{s}\right\} \subseteq H$ to be of maximal size subject to:

(i) $1 \in \Lambda$ and the $h_{i}$ are in distinct right cosets of $W$ in $H$;

(ii) $K=J^{h_{1}} J^{h_{2}} \ldots J^{h_{s}} \neq 0$.

Now let $x \in H$ and suppose that $K^{x} K \neq 0$. Then, by considering the right cosets of $W$, we must have $W \Lambda x=W \Lambda$. Indeed, if this were not the case then, for some $i, h_{i} x$ would be in a new right coset of $W$. We would then have

$$
J^{h_{i} x} J^{h_{1}} J^{h_{2}} \cdots J^{h_{s}} \supseteq K^{x} K \neq 0
$$

contradicting the maximality of $\Lambda$. Since $1 \in \Lambda$ and $W \Lambda x=W \Lambda$, this implies that $h_{i} x \in W$ for some $i$. Furthermore $J^{h_{i} x} J \supseteq K^{x} K \neq 0$ so, by hypothesis since $h_{i} x \in W$, we must have $h_{i} x \in \bigcup_{1}^{n} w_{k} H_{k}$.

We have therefore shown that $K^{x} K \neq 0$ implies that $x \in h_{i}^{-1}\left(\cup_{1}^{n} w_{k} H_{k}\right)$ for some $i$. Equivalently, we have

$$
K^{h} K=0 \text { for all } h \in H \backslash \bigcup_{i, k} h_{i}^{-1} w_{k} H_{k} .
$$

We can now apply Lemma 2.1 to this situation to obtain a nonzero product

$$
I=K^{v_{1}} K^{y_{2}} \cdots K^{y_{t}} \neq 0
$$

with some $y_{j}=1$ and a subgroup $L$ of $H$ with $\left|L: L \cap H_{k}\right|<\infty$ for some $k$ and $I^{h} I=0$ for all $h \in H \backslash L$. Since $1 \in \Lambda, I$ is an appropriate product of $H$-conjugates of $J$, thereby completing the proof.

Again there are three other forms of this lemma which are equally valid. We close this section with some definitions and minor observations. We assume in the remaining three lemmas that $G$ strongly permutes the ideals of $R$.

LEMMA 2.3. Let I be an ideal of $R$.

(i) $I^{G}=\sum_{x \in G} I^{x}$ is the smallest $G$-invariant ideal of $R$ containing $I$.

(ii) If $I$ is $G$-invariant, then so are $\underline{r}_{R}(I)$ and $l_{R}(I)$.

Proof. (i) is clear since the action of $G$ preserves arbitrary sums and (ii) follows from the formula $(I J)^{x}=I^{x} J^{x}$.

Now let $I$ be a $G$-invariant ideal of $R$. Then $I$ is said to be $G$-nilpotent-free if $I$ contains no nonzero $G$-invariant nilpotent ideal of $R$. Similarly $I$ is said to be $G$-annihilator-free if for all nonzero $G$-invariant ideals $A, B \subseteq I$ we have $A B \neq 0$. Obviously the latter property implies the former. When $I=R$ these conditions assert precisely that $R$ is $G$-semiprime or $G$-prime, respectively.

LEMMA 2.4. Let I be a G-invariant ideal of $R$.

(i) If I is G-nilpotent-free then $\underline{r}_{R}(I)=\underline{r}_{R}\left(I^{2}\right)$.

(ii) If $H$ is a subgroup of $G$ of finite index, then $I$ is $G$-nilpotent free if and only if it is $H$-nilpotent-free. 
Proof. (i) Obviously $\underline{r}_{R}(I) \subseteq \underline{r}_{R}\left(I^{2}\right)=J$ and these are $G$-invariant ideals of $R$. Since $I^{2} J=0$, we see that $I J$ is a $G$-invariant ideal contained in $I$ with $(I J)^{2} \subseteq I$. (IJ) $=0$. Thus $I J=0$ and $J \subseteq \underline{r}_{R}(I)$.

(ii) If $I$ is $H$-nilpotent-free then it is obviously $G$-nilpotent-free. Conversely suppose $I$ is $G$-nilpotent-free and let $J$ be an $H$-invariant nilpotent ideal of $R$ contained in $I$. Then since $|G: H|<\infty, J^{G}$ is a finite sum of the nilpotent ideals $J^{x}$ and hence $J^{G}$ is nilpotent. Thus $J^{G}=0$ and $J=0$.

Lemma 2.5. Let $H$ be a subgroup of $G$ and let $I$ be an ideal of $R$. Suppose that $I^{x} I=0$ for all $x \in G \backslash H$.

(i) $I^{H}$ is an $H$-invariant ideal of $R$ with

$$
\left(I^{H}\right)^{x}\left(I^{H}\right)=0 \quad \text { for all } x \in G \backslash H .
$$

(ii) Let $I \subseteq J$ with $I$ an $H$-invariant ideal and with $J$ a G-invariant ideal. If $J$ is G-nilpotent-free (or G-annihilator-free), then I is $\mathrm{H}$-nilpotent-free (or $\mathrm{H}$-annihilatorfree, respectively).

Proof. (i) If $a, b \in H$ and $x \in G \backslash H$, then $I^{a x} I^{b}=\left(I^{a x b^{-1}} I\right)^{b}=0$ since $a x b^{-1} \notin$ $H$.

(ii) Let $A$ and $B$ be $H$-invariant ideals of $R$ contained in $I$ with $A B=0$. By considering the cases $x \in H$ and $x \in G \backslash H$ separately, we see that $A^{x} B=0$ for all $x \in G$. It then follows that $A^{G}$ and $B^{G}$ are $G$-invariant ideals of $R$ contained in $J$ with $A^{G} B^{G}=0$. If $J$ is $G$-annihilator-free then $A^{G}$ or $B^{G}$ is zero and hence $I$ is $H$-annihilator-free. Finally by taking $A=B$ we obtain the analogous result for nilpotent-free ideals.

3. $\Delta$-methods. Let $R * G$ be a strongly $G$-graded ring. By Lemma $1.2, G$ strongly permutes the ideals of $R$. Therefore all the results and definitions of $\S 2$ apply here. We will use this observation without further comment.

If $H$ is a subgroup of $G$, then there is a natural projection map $\pi_{H}: R * G \rightarrow R * H$ given by

$$
\pi_{H}\left(\sum_{x \in G} \alpha_{x}\right)=\sum_{x \in H} \alpha_{x} .
$$

This is easily seen to be an $(R * H, R * H)$-bimodule homomorphism.

Again, if $H \subseteq G$ then the almost centralizer of $H$ in $G$ is defined by

$$
\mathbf{D}_{G}(H)=\left\{x \in G|| H: \mathbf{C}_{H}(x) \mid<\infty\right\} .
$$

It is clear that $\mathbf{D}_{G}(H)$ is a subgroup of $G$ normalized by $H$. Furthermore, $H \cap$ $\mathbf{D}_{G}(H)=\mathbf{D}_{H}(H)=\Delta(H)$ where $\Delta(H)$ denotes the f.c. (finite conjugate) center of $H$.

The main result of this section is

Proposition 3.1. Let $R * G$ be a strongly $G$-graded ring and assume that the base ring $R$ is $G$-semiprime. Suppose that $A$ and $B$ are nonzero ideals of $R * G$ with $A B=0$. 
Then there exists a subgroup $H \subseteq G$, a nonzero $H$-invariant ideal $I$ of $R$ and an element $\beta \in B$ such that:

(i) $I^{x} I=0$ for all $x \in G \backslash H$;

(ii) $I \pi_{\Delta}(A) \neq 0, I \pi_{\Delta}(\beta) \neq 0$ where $\Delta=\Delta(H)$;

(iii) $I \pi_{\Delta}(A) \cdot I \beta=0$.

The above conditions motivate the following definition. Suppose $A$ and $B$ are nonzero ideals of $R * G$ with $A B=0$. We say that the 4-tuple $(H, D, I, \beta)$ is a form for $A, B$ if:

(i) $H \subseteq G, D=\mathbf{D}_{G}(H)$;

(ii) $I$ is an $H$-invariant ideal of $R$ with $I^{x} I=0$ for all $x \in G \backslash H$;

(iii) $\beta \in B, I \beta \neq 0, I A \neq 0$.

For the remainder of this section we assume the hypotheses of Proposition 3.1. The proof of the latter proceeds in a sequence of lemmas.

LEMMA 3.2. Forms exist.

Proof. Take $H=G, D=\Delta(G)$ and $I=R$. Since $A, B \neq 0$ and $1 \in R$ we have $I A \neq 0, I \beta \neq 0$ for any $\beta \in B \backslash 0$.

We define $n=(H, D, I, \beta)^{\#}$, the size of the form, to be the number of right $D$-cosets meeting $\operatorname{Supp} \beta$. We now assume for the rest of this argument that $(H, D, I, \beta)$ is a form whose size $n$ is minimal. In the next lemma we make a slight change in $\beta$. Afterwards, no additional changes in the form will be made. Set $\Delta=\Delta(H)$.

LEMMA 3.3. With the above notation:

(i) $I$ is $H$-nilpotent-free and $\underline{r}_{R * G}(I)=\underline{r}_{R * G}\left(I^{2}\right)$;

(ii) $I \pi_{\Delta}(A) \neq 0$ and we may assume that $I \pi_{\Delta}(\beta) \neq 0$;

(iii) if $\gamma \in R * D$, then $I \gamma \beta=0$ if and only if $I \gamma \pi_{D}(\beta)=0$.

Proof. (i) By assumption, $R$ is $G$-semiprime. Since $I$ is $H$-invariant and $I^{x} I=0$ for all $x \in G \backslash H$, it follows from Lemma 2.5(ii) that $I$ is $H$-nilpotent-free. Hence, by Lemma 2.4(i), $\underline{r}_{R}(I)=\underline{r}_{R}\left(I^{2}\right)$. Finally, $\underline{r}_{R * G}(I) \subseteq \underline{r}_{R} * G\left(I^{2}\right)$ and suppose $\gamma=\sum \gamma_{x}$ is contained in $\underline{r}_{R * G}\left(I^{2}\right)$. Then for all $x$ we have $I^{2} \gamma_{x}=0$ so $\gamma_{x} R_{x^{-1}} \subseteq \underline{r}_{R}\left(I^{2}\right)=\underline{r}_{R}(I)$. This yields $I \gamma_{x} R_{x^{-1}}=0$ so $I \gamma_{x}=0$ and hence $\gamma \in \underline{r}_{R * G}(I)$.

(ii) Since $I A \neq 0$ we have $I A R_{x} \neq 0$ for all $x$. Thus since $A R_{x} \subseteq A$ it follows immediately that $I \pi_{\Delta}(A) \neq 0$. Now write $\beta=\sum \beta_{x}$. Since $I \beta \neq 0$ we have $I \beta_{x} \neq 0$ for some $x \in G$. Thus $I \beta_{x} R_{x^{-1}} \neq 0$ and we can choose $\sigma_{x^{-1}} \in R_{x^{-1}}$ with $I \beta_{x} \sigma_{x^{-1}} \neq 0$. It is now clear that $\left(H, D, I, \beta \sigma_{x^{-1}}\right)$ is also a form with the additional property that $I \pi_{\Delta}\left(\beta \sigma_{x^{-1}}\right) \neq 0$. Furthermore, we have

$$
\left(H, D, I, \beta \sigma_{x^{-1}}\right)^{\#} \leqslant(H, D, I, \beta)^{\#}
$$

so $\left(H, D, I, \beta \sigma_{x^{-1}}\right)$ also has minimal size $n$. We now replace $\beta$ by $\beta \sigma_{x^{-1}}$ for the remainder of the proof of the proposition, or equivalently we can assume that $I \pi_{\Delta}(\beta) \neq 0$. This implies in particular that $\pi_{\Delta}(\beta) \neq 0$ and hence $\pi_{D}(\beta) \neq 0$ since $D \supseteq \Delta$. 
(iii) If $I \gamma \beta=0$, then applying $\pi_{D}$ yields $I \gamma \pi_{D}(\beta)=0$. Conversely suppose $I \gamma \pi_{D}(\beta)=0$. Then for any $s \in I$ we have $s \gamma \beta \in B$ and Supp $s \gamma \beta$ meets less than $n$ right cosets of $D$ since $\gamma \in R * D, \pi_{D}(s \gamma \beta)=0$ but $\pi_{D}(\beta) \neq 0$. By definition of $n$, this implies that $(H, D, I, s \gamma \beta)$ is not a form. Thus $I s \gamma \beta=0$ for all $s \in I$ so $I^{2} \gamma \beta=0$. We conclude from (i) above that $I \gamma \beta=0$.

It follows from the above that $H, I$ and $\beta$ satisfy (i) and (ii) of the conclusion of Proposition 3.1. If in addition they satisfy condition (iii), then the result is proved. Thus we will assume throughout the remainder of this section that $I \pi_{\Delta}(A) \cdot I \beta \neq 0$ and we derive a contradiction.

LEMMA 3.4. With the above assumption, there exists $W$ a subgroup of $H$ of finite index, $\alpha=\sum \alpha_{x} \in A \cap(R * H)$ and $d \in \operatorname{Supp} \pi_{D}(\alpha)$ such that:

(i) $W$ centralizes $\operatorname{Supp} \pi_{D}(\alpha)$ and $\operatorname{Supp} \pi_{D}(\beta)$;

(ii) for some $u \in W$

$$
I \pi_{D}(\alpha) \beta \supseteq\left(I \alpha_{d} R_{d^{-1}}\right)^{u} \pi_{D}(\alpha) \beta \neq 0 ;
$$

(iii) for all $y \in W$

$$
I \alpha_{d} R_{d^{-1} y} \pi_{D}(\alpha) \pi_{D}(\beta)=I \pi_{D}(\alpha) R_{d^{-1} y} \alpha_{d} \pi_{D}(\beta) .
$$

Proof. (i) By assumption $I \pi_{\Delta}(A) \cdot I \beta \neq 0$ and hence, since $\underline{r}_{R * G}(I)=\underline{r}_{R * G}\left(I^{2}\right)$ we also have

$$
I \pi_{\Delta}(I A I) \beta=I^{2} \pi_{\Delta}(A) \cdot I \beta \neq 0 .
$$

Thus there exists $\alpha \in I A I \subseteq A$ with $I \pi_{\Delta}(\alpha) \beta \neq 0$. Observe that $\alpha \in I(R * G) I \subseteq$ $R * H$, by Lemma 1.4(ii). Thus since $D \cap H=\Delta$ we have $\pi_{D}(\alpha)=\pi_{\Delta}(\alpha)$ and $I \pi_{D}(\alpha) \beta \neq 0$.

We can now assume that $\alpha$ is chosen so that $\left|\operatorname{Supp} \pi_{D}(\alpha)\right|$ is minimal subject to $\alpha \in A \cap(R * H)$ and $I \pi_{D}(\alpha) \beta \neq 0$. Let $W$ be the intersection of the centralizers in $H$ of the elements of $\operatorname{Supp} \pi_{D}(\alpha)$ and of $\operatorname{Supp} \pi_{D}(\beta)$. Since $\operatorname{Supp} \pi_{D}(\alpha) \cup$ Supp $\pi_{D}(\beta)$ is a finite subset of $D=\mathbf{D}_{G}(H)$, it is clear that $|H: W|<\infty$. Note that $I$ is an $H$-nilpotent-free ideal, by Lemma 3.3(i), and hence it is also $W$-nilpotent-free by Lemma 2.4(ii). This completes the proof of (i).

(ii) This part does not use the minimal nature of Supp $\pi_{D}(\alpha)$. Set $\gamma=\pi_{D}(\alpha) \beta$ and write $\alpha=\sum_{x \in G} \alpha_{x}, \beta=\sum_{x \in G} \beta_{x}$ and $\gamma=\sum_{x \in G} \gamma_{x}$. Then $\pi_{D}(\alpha)=\sum_{d \in D} \alpha_{d}$ and we let $J$ be the $W$-invariant ideal of $R$ given by $J=\sum_{d \in D}\left(R \alpha_{d} R_{d^{-1}}\right)^{W}$. Notice that for all $d \in D, y \in G$ we have

$$
\alpha_{d} \beta_{y} R_{y^{-1} d^{-1}} \subseteq R \alpha_{d} R_{d^{-1}} \cdot R_{d} \beta_{y} R_{y^{-1} d^{-1}} \subseteq J \cdot R=J
$$

and from this it follows that $\gamma_{x} R_{x^{-1}} \subseteq J$ for all $x \in G$. Now suppose that $I J \gamma=0$. Then $I J \gamma_{x} R_{x^{-1}}=0$ so $\gamma_{x} R_{x^{-1}} \subseteq \underline{r}_{R}(I J)$ and hence, by the above,

$$
I \gamma_{x} R_{x^{-1}} \subseteq I J \cap \underline{r}_{R}(I J)=0
$$

since the latter is a $W$-invariant nilpotent ideal contained in $I$. This yields $I \gamma_{x}=0$ and therefore $I \pi_{D}(\alpha) \beta=I \gamma=0$, a contradiction by the choice of $\alpha$. Thus we have $I J \pi_{D}(\alpha) \beta \neq 0$ and hence, by definition of $J$, there exist $d \in \operatorname{Supp} \pi_{D}(\alpha)$ and $u \in W$ with $I\left(R \alpha_{d} R_{d^{-1}}\right)^{u} \pi_{D}(\alpha) \beta \neq 0$. Since $u \in W \subseteq H$ and $I$ is $H$-invariant, this part is proved. 
(iii) Let $y \in W$ and choose any $a_{y^{-1}} \in R_{y^{-1}}$ and $b_{d^{-1} y} \in R_{d^{-1} y}$. We study the element

$$
\gamma=a_{y^{-1}} \alpha_{d} b_{d^{-1} y} \alpha-a_{y^{-1}} \alpha b_{d^{-1} y} \alpha_{d} .
$$

Clearly $\gamma \in(R * H) \cap A$ and since $\alpha=\sum_{x \in G} \alpha_{x}$ we have $\gamma=\sum_{x \in G} \sigma(x)$ where

$$
\sigma(x)=a_{y^{-1}} \alpha_{d} b_{d^{-1} y} \alpha_{x}-a_{y^{-1}} \alpha_{x} b_{d^{-1} y} \alpha_{d} .
$$

Observe that, since $y$ centralizes $d$, the summands in $\sigma(x)$ have grades $x$ and $y^{-1} x y$, respectively. In particular, if $x \notin D$ then neither of these grades is in $D$ since $y \in H$ normalizes $D$. On the other hand, if $x \in D$ then $x \in \operatorname{Supp} \pi_{D}(\alpha)$ so $y$ commutes with $x$ and hence both these summands have grade $x \in D$. It follows that $\pi_{D}(\gamma)=$ $\sum_{x \in D^{\sigma}} \sigma(x)$ and that $\left|\operatorname{Supp} \pi_{D}(\gamma)\right| \leqslant\left|\operatorname{Supp} \pi_{D}(\alpha)\right|$. In fact, this inequality is strict since clearly $\sigma(d)=0$.

The minimality of $\left|\operatorname{Supp} \pi_{D}(\alpha)\right|$ now implies that $I \pi_{D}(\gamma) \beta=0$ and hence, by applying $\pi_{D}$, that $I \pi_{D}(\gamma) \pi_{D}(\beta)=0$. Now as we observed above, $\pi_{D}(\gamma)$ comes precisely from the $D$-homogeneous components of $\alpha$ so we have

$$
\pi_{D}(\gamma)=a_{y^{-1}} \alpha_{d} b_{d^{-1} y} \pi_{D}(\alpha)-a_{y^{-1}} \pi_{D}(\alpha) b_{d^{-1} y} \alpha_{d}
$$

and hence

$$
I a_{y^{-1}}\left(\alpha_{d} b_{d^{-1} y} \pi_{D}(\alpha)-\pi_{D}(\alpha) b_{d^{-1} y} \alpha_{d}\right) \pi_{D}(\beta)=0 .
$$

Notice that this formula holds for all $a_{y^{-1}} \in R_{y^{-1}}$ and that $I R_{y^{-1}}=R_{y^{-1}} I$ since $I$ is $H$-invariant. We can therefore cancel the $R_{y^{-1}}$ factor and obtain

$$
I \alpha_{d} b_{d^{-1} y} \pi_{D}(\alpha) \pi_{D}(\beta)=I \pi_{D}(\alpha) b_{d^{-1} y} \alpha_{d} \pi_{D}(\beta)
$$

and since this holds for all $b_{d^{-1} y} \in R_{d^{-1} y}$, the lemma is proved.

The following $\Delta$-lemma is a variant of [8, Lemma 1.5]. Fix $\alpha=\sum \alpha_{x}, d, W$ and $u$ as in the preceding result for the remainder of this argument.

LEMMA 3.5. With the above notation,

$$
\left(I \alpha_{d} R_{d^{-1}}\right)^{y} \cdot \pi_{D}(\alpha) \beta=0
$$

for all $y \in W \backslash \bigcup_{1}^{t} x_{i} H_{i}$. Here $\bigcup_{1}^{t} x_{i} H_{i}$ is a fixed finite union of left cosets of the subgroups $H_{i}$ and each $H_{i}$ is the centralizer in $W$ of some element of $(\operatorname{Supp} \beta) \backslash D$.

Proof. We freely use the fact that $I$ is $H$-invariant and, in particular, that $R_{h} I=I R_{h}$ for all $h \in H$.

Let $y \in W$ and suppose that

$$
\left(I \alpha_{d} R_{d^{-1}}\right)^{y} \pi_{D}(\alpha) \beta \neq 0 .
$$

Then

$$
I\left(R \alpha_{d} R_{d^{-1}}\right)^{y} \pi_{D}(\alpha) \cdot \beta \neq 0
$$

so Lemma 3.3(iii) yields

$$
I\left(R \alpha_{d} R_{d^{-1}}\right)^{v} \pi_{D}(\alpha) \pi_{D}(\beta) \neq 0 .
$$


Thus we have

$$
I \alpha_{d} R_{d^{-1} y} \pi_{D}(\alpha) \pi_{D}(\beta) \neq 0
$$

so Lemma 3.4(iii) yields

$$
I \pi_{D}(\alpha) R_{d^{-1} y} \alpha_{d} \pi_{D}(\beta) \neq 0
$$

and therefore finally

$$
I \pi_{D}(\alpha) R_{d^{-1} y} \alpha_{d} \pi_{D}(\beta) R_{y^{-1}} \neq 0 .
$$

Write $\alpha=\pi_{D}(\alpha)+\tilde{\alpha}$ and $\beta=\pi_{D}(\beta)+\tilde{\beta}$. Thus since $I \alpha R_{d^{-1} y} \alpha_{d} \beta R_{y^{-1}} \subseteq A B=0$ we have

$$
I\left(\pi_{D}(\alpha)+\tilde{\alpha}\right) R_{d^{-1} y^{\prime}} \alpha_{d}\left(\pi_{D}(\beta)+\tilde{\beta}\right) R_{y^{-1}}=0 .
$$

We now consider the supports of each of the four summands obtained from the above expression to see how cancellation can occur. Observe that $y \in W$ so $y$ normalizes $D$ and centralizes $d \in \operatorname{Supp} \pi_{D}(\alpha)$. In particular, we have $R_{d^{-1} y} \alpha_{d} \subseteq R_{y}$ and from this it follows easily that the sets

$$
I \pi_{D}(\alpha) R_{d^{-1} y} \alpha_{d} \tilde{\beta} R_{y^{-1}} \text { and } I \tilde{\alpha} R_{d^{-1} y} \alpha_{d} \pi_{D}(\beta) R_{y^{-1}}
$$

have supports disjoint from $D$. On the other hand,

$$
0 \neq I \pi_{D}(\alpha) R_{d^{-1} y} \alpha_{d} \pi_{D}(\beta) R_{y^{-1}} \subseteq R * D
$$

by the work of the preceding paragraph so it follows that this expression must be cancelled by terms from the fourth summand

$$
I \tilde{\alpha} R_{d^{-1}{ }} \alpha_{d} \tilde{\beta} R_{y^{-1}} .
$$

In particular, the latter two summands must have a support element in common.

Thus there exist $f \in \operatorname{Supp} \tilde{\alpha}, g \in \operatorname{Supp} \tilde{\beta}, a \in \operatorname{Supp} \pi_{D}(\alpha)$ and $b \in \operatorname{Supp} \pi_{D}(\beta)$ with $a y b y^{-1}=f y g y^{-1}$. Since $y \in W$ centralizes $b \in \operatorname{Supp} \pi_{D}(\beta)$, this yields $g^{y^{-1}}=$ $y g y^{-1}=f^{-1} a b$ so $y \in x \mathbf{C}_{W}(g)$, some fixed left coset of $\mathbf{C}_{W}(g)$ depending only on the finitely many parameters $f, g, a, b$. Since $g \in \operatorname{Supp} \tilde{\beta}=(\operatorname{Supp} \beta) \backslash D$, the lemma is proved.

We remark that the truncation from $\beta$ to $\pi_{D}(\beta)$, using Lemma 3.3(iii), in the above argument is crucial. Otherwise the subgroups $H_{i}$ turn out to be centralizers of elements in $(\operatorname{Supp} \alpha) \backslash D$.

LEMMA 3.6. Contradiction.

Proof. We use the notation of the preceding two lemmas and we set $\gamma=\pi_{D}(\alpha) \beta$ $=\sum_{x \in G} \gamma_{x}$. Then by Lemma 3.4(ii), there exists $x \in \operatorname{Supp} \gamma$ with $\left(I \alpha_{d} R_{d^{-1}}\right)^{u} \gamma_{x} \neq 0$ and hence

$$
J=\left(I \alpha_{d} R_{d^{-1}}\right)^{u} \gamma_{x} R_{x^{-1}}
$$

is a nonzero ideal of $R$ contained in $I$ since $I$ is $H$-invariant and $u \in W \subseteq H$. Furthermore, since $J \subseteq\left(I \alpha_{d} R_{d^{-1}}\right)^{u}$ we have $J^{u^{-1}} \subseteq I \alpha_{d} R_{d^{-1}}$ and hence $J^{u^{-1} y} \subseteq$ $\left(I \alpha_{d} R_{d^{-1}}\right)^{y}$ for all $y \in W$. 


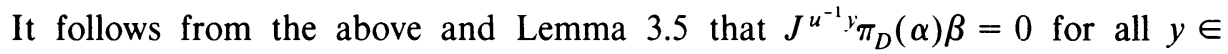
$W \backslash \bigcup_{1}^{t} x_{i} H_{i}$ or equivalently that $J^{v} \pi_{D}(\alpha) \beta=0$ for all $y \in W \backslash \bigcup_{1}^{t} u^{-1} x_{i} H_{i}$ since $u \in W$. In particular, $J^{y} \gamma_{x}=0$ for these $y$ so $J^{y}\left(R \gamma_{x} R_{x^{-1}}\right)=0$ and hence $J^{y} J=0$. Since $|H: W|<\infty$, Lemma 2.2 applies and there exists a subgroup $L$ of $H$ and a nonzero product $K=J^{v_{1}} J^{v_{2}} \cdots J^{v_{s}}$ such that $K^{h} K=0$ for all $h \in H \backslash L$. Furthermore, $v_{i}=1$ for some $i$ so $K \subseteq J \subseteq I$ and $\left|L: L \cap H_{k}\right|<\infty$ for some $k>0$.

We claim that $\left(L, \mathbf{D}_{G}(L), K^{L}, \pi_{D}(\alpha) \beta\right)$ is also a form. To start with, we have $K^{h} K=0$ for all $h \in H \backslash L$ and $K \subseteq I$ so $K^{g} K=0$ for all $g \in G \backslash L$. Thus, by Lemma 2.5(i), $K^{L}$ is a nonzero $L$-invariant ideal of $R$ with $\left(K^{L}\right)^{g} K^{L}=0$ for all $g \in G \backslash L$. Furthermore, since $R$ is $G$-semiprime, Lemma 2.5 (ii) implies that $K^{L}$ is $L$-nilpotent-free and in particular $\left(K^{L}\right)^{2} \neq 0$. Suppose $K^{L} \gamma=K^{L} \pi_{D}(\alpha) \beta=0$. Then $K^{L} \gamma_{x}=0$ so $K^{L}\left(R \gamma_{x} R_{x^{-1}}\right)=0$ and hence $K^{L} J=0$. But $\underline{r}_{R}\left(K^{L}\right)$ is $L$-invariant, by Lemma 2.3(ii), so this yields $K^{L} J^{L}=0$ and hence, since $K \subseteq J$, we have $\left(K^{L}\right)^{2}=0$, a contradiction. Thus $K^{L} \pi_{D}(\alpha) \beta \neq 0$. Furthermore, this implies that $K^{L} \pi_{D}(\alpha) \neq 0$ so since $\alpha \in A$ we have $K^{L} A \neq 0$. Thus since $\pi_{D}(\alpha) \beta \in B,\left(L, \mathbf{D}_{G}(L), K^{L}, \pi_{D}(\alpha) \beta\right)$ is indeed a form.

It remains to compute the size of this new form. Since $H \supseteq L$ we have $\mathbf{D}_{G}(L) \supseteq$ $\mathbf{D}_{G}(H)=D$. Thus since $\pi_{D}(\alpha) \in R * D$, it is clear that Supp $\pi_{D}(\alpha) \beta$ meets at most $n$ right cosets of $D$ and hence at most $n$ right cosets of $\mathbf{D}_{G}(L)$. But observe that $\left|L: L \cap H_{k}\right|<\infty$ and that $H_{k}=\mathrm{C}_{W}(g)$ for some $g \in(\operatorname{Supp} \beta) \backslash D$. Thus $\left|L: \mathbf{C}_{L}(g)\right|<\infty$ so $g \in \mathbf{D}_{G}(L)$ and, in fact, $D g \subseteq \mathbf{D}_{G}(L)$. Since $\pi_{D}(\beta) \neq 0$, the two $D$-cosets $D$ and $D g$ which meet elements in $\operatorname{Supp} \beta$ merge to the single coset $\mathbf{D}_{G}(L)$ and therefore Supp $\pi_{D}(\alpha) \beta$ meets less than $n$ right cosets of $\mathbf{D}_{G}(L)$. In other words,

$$
\left(L, \mathbf{D}_{G}(L), K^{L}, \pi_{D}(\alpha) \beta\right)^{\#}<(H, D, I, \beta)^{\#},
$$

contradicting the minimal nature of $(H, D, I, \beta)$.

As we observed previously, the contradiction of Lemma 3.6 is based on the assumption that $I \pi_{\Delta}(A) \cdot I \beta \neq 0$. Thus $I \pi_{\Delta}(A) \cdot I \beta=0$ and Proposition 3.1 is proved.

4. The main theorem. The goal of this section is to complete the proof of Theorem 1.3 and to obtain its corollaries on primeness. As we will see, the interesting work has already been done and only a series of routine reductions remain. We first require a few simple observations.

Suppose $A$ is a nonzero ideal of the strongly $W$-graded ring $R * W$ and let $N \triangleleft W$. Then we denote by $\min _{N} A$ the span of all elements $\alpha \neq 0$ of $A$ whose support meets the minimal number of cosets of $N$.

LEMma 4.1. Let $R * G$ be given, let $H \subseteq G$ and let $N \triangleleft W$ be subgroups of $G$ normalized by $H$. If $A$ is a nonzero $H$-invariant ideal of $R * W$, then:

(i) $\min _{N} A$ is a nonzero $H$-invariant ideal of $R * W$.

(ii) $\pi_{N}(A)$ is a nonzero $H$-invariant ideal of $R * N$.

Proof. (i) By definition, $\min _{N} A$ is nonzero and let $\alpha \in A$ be any generator of this set. If $w \in W$, then $\operatorname{Supp} \alpha R_{w} \subseteq(\operatorname{Supp} \alpha) w$ and $\operatorname{Supp} R_{w} \alpha \subseteq w(\operatorname{Supp} \alpha)$ so, since $N \triangleleft W$, it follows that $\min _{N} A$ is an ideal of $R * W$. Finally, if $h \in H$, then 
Supp $R_{h^{-1}} \alpha R_{h} \subseteq h^{-1}(\operatorname{Supp} \alpha) h$ so, since $A$ is $H$-invariant and $N$ is normalized by $H$, we see that $\min _{N} A$ is $H$-invariant.

(ii) We know that $\pi_{N}$ is an $(R * N, R * N)$-bimodule homomorphism so $\pi_{N}(A)$ is an ideal of $R * N$. Next if $\alpha \in A$ and $h \in H$ then, since $H$ normalizes $N$, we have $\pi_{N}\left(R_{h^{-1}} \alpha R_{h}\right)=R_{h^{-1}} \pi_{N}(\alpha) R_{h}$ and therefore $\pi_{N}(A)$ is $H$-invariant. Finally choose $0 \neq \alpha=\sum_{x \in W} \alpha_{x} \in A$. If $x \in \operatorname{Supp} \alpha$, then $\alpha R_{x^{-1}} \subseteq A$ and clearly $\pi_{N}\left(\alpha R_{x^{-1}}\right) \neq 0$.

LemMA 4.2. Let $R * W$ be given, let $N \triangleleft W$ and assume that $W / N$ is a unique product group (for example, an ordered group). If $A$ and $B$ are nonzero ideals of $R * W$ with $A B=0$, then

$$
\pi_{N}\left(\min _{N} A\right) \cdot \pi_{N}\left(\min _{N} B\right)=0 .
$$

Proof. If $\alpha=\sum_{w \in W} \alpha_{w}$, then by summing the homogeneous components over each coset of $N$, we can write $\alpha$ uniquely as $\alpha=\sum_{\bar{x} \in W / N} \alpha_{\bar{x}}$. In this way, $R * W$ becomes a strongly $(W / N)$-graded ring and we use this notation here. In particular, $\pi_{N}(\alpha)=\alpha_{\overline{1}}^{-}$

Let $\alpha=\sum \alpha_{\bar{x}}$ and $\beta=\sum \beta_{\bar{y}}$ be generators of $\min _{N} A$ and $\min _{N} B$, respectively. Since $W / N$ is a unique product group, we can let $\bar{x}_{0} \bar{y}_{0}$ be a unique product element in $(\operatorname{Supp} \alpha) N / N \cdot(\operatorname{Supp} \beta) N / N$. From $\alpha \beta=0$ we deduce that $\alpha_{\bar{x}_{0}} \beta_{\overline{y_{0}}}=0$. Then $\alpha \beta_{\bar{y}_{0}} \in A$ and the support of this element meets less cosets of $N$ than does Supp $\alpha$. Hence, by the minimal nature of $\alpha$, we have $\alpha \beta_{\bar{y}_{0}}=0$ and therefore $\alpha_{\bar{x}} \beta_{\bar{y}_{0}}=0$ for all $\bar{x} \in W / N$. Similarly $\alpha_{\bar{x}} \beta \in B$ and the support of this element meets less cosets of $N$ than does $\operatorname{Supp} \beta$. Thus $\alpha_{\bar{x}} \beta=0$ so $\alpha_{\bar{x}} \beta_{\bar{y}}=0$ for all $\bar{x}, \bar{y} \in W / N$. In particular, $\pi_{N}(\alpha) \pi_{N}(\beta)=\alpha_{1}^{-} \beta_{\overline{1}}=0$.

We can now offer the

Proof of Theorem 1.3 (HARD Direction). We assume that $R * G$ is a strongly $G$-graded ring and that $A$ and $B$ are nonzero ideals of $R * G$ with $A B=0$. Suppose first that $R$ is not $G$-semiprime. Then there exists a nonzero $G$-invariant ideal $\tilde{A}$ of $R$ with $\tilde{A}^{2}=0$. The result now follows with $H=G, I=R, N=\langle 1\rangle$ and $\tilde{B}=\tilde{A}$.

We can therefore assume that $R$ is $G$-semiprime so Proposition 3.1 applies. Thus there exist $H \subseteq G, I$ a nonzero $H$-invariant ideal of $R$, and $\beta \in B$ such that:

(i) $I^{x} I=0$ for all $x \in G \backslash H$;

(ii) $I \pi_{\Delta}(A) \neq 0, I \pi_{\Delta}(\beta) \neq 0$ where $\Delta=\Delta(H)$;

(iii) $I \pi_{\Delta}(A) \cdot I \beta=0$.

We have therefore found an appropriate $H$ and $I$. It remains to find $N, \tilde{A}$ and $\tilde{B}$.

Set $A_{1}=I \pi_{\Delta}(A)$ and $B_{1}=I \cdot\left((R * \Delta) \pi_{\Delta}(\beta)(R * \Delta)\right)^{H}$. By Lemma 4.1 and (ii) above, $A_{1}$ is a nonzero $H$-invariant ideal of $R * \Delta$. Since $I \pi_{\Delta}(A) \cdot I \beta=0$ we have $I \pi_{\Delta}(A) \cdot I \pi_{\Delta}(\beta)=0$ and, by (ii) again, it follows that $B_{1}$ is a nonzero $H$-invariant ideal of $R * \Delta$ with $A_{1} B_{1}=0$. Note that $A_{1}, B_{1} \subseteq I(R * \Delta)$ and, since $I \pi_{\Delta}(\beta) \subseteq$ $I \pi_{\Delta}(B)$, we have $B_{1} \subseteq I \pi_{\Delta}(B)$.

Since $A_{1}$ and $B_{1}$ are nonzero and since every element of $\Delta(H)$ has only finitely many $H$-conjugates, it is clear that there exists a normal subgroup $W$ of $H$ with $W \subseteq \Delta, W$ finitely generated and $A_{2}=A_{1} \cap(R * W)$ and $B_{2}=B_{1} \cap(R * W)$ both nonzero. Certainly $A_{2}$ and $B_{2}$ are nonzero $H$-invariant ideals of $R * W$ contained in $I(R * W)$ with $A_{2} B_{2}=0$. 
Now $W$ is a finitely generated subgroup of $\Delta(H)$ so by [13, Lemma 4.1.5(iii)], $W$ has a finite characteristic subgroup $N$ with $W / N$ torsion-free abelian. Set $A_{3}=$ $\min _{N} A_{2}, A_{4}=\pi_{N}\left(A_{3}\right), B_{3}=\min _{N} B_{2}$ and $B_{4}=\pi_{N}\left(B_{3}\right)$. Since $N \triangleleft H$, Lemma 4.1 implies that $A_{3}$ and $B_{3}$ are nonzero $H$-invariant ideals of $R * W$ contained in $I(R * W)$ and then that $A_{4}$ and $B_{4}$ are nonzero $H$-invariant ideals of $R * N$ contained in $I(R * N)$. Furthermore, since $W / N$ is an ordered group and $A_{2} B_{2}=0$, Lemma 4.2 implies that $A_{4} B_{4}=0$. For general $A$ and $B$, the result now follows by taking $N$ as above, $\tilde{A}=A_{4}$ and $\tilde{B}=B_{4}$.

Finally if $A=B$, then since

$$
B_{1} \subseteq I \pi_{\Delta}(B)=I \pi_{\Delta}(A)=A_{1}
$$

we have $B_{1}^{2}=0$. It then follows as above that $B_{i}^{2}=0$ for all $i$ so we can take $\tilde{A}=\tilde{B}=B_{4}$. This completes the proof.

We now consider a few consequences. The first is really just a reformulation and slight extension of Theorem 1.3. To avoid trivialities we will assume that $R$ is $G$-semiprime. We start with some definitions.

Let $G$ strongly permute the ideals of $R$ and let $I$ be an ideal of $R$. Then we denote by

$$
G_{I}=\left\{x \in G \mid I^{x}=I\right\}
$$

the stabilizer of $I$ in $G$. The nonzero ideal $I$ is said to be a trivial intersection ideal if for all $x \in G$ either $I^{x}=I$ or $I^{x} \cap I=0$. Note that $I^{x} \cap I=0$ implies $I^{x} I=0$.

LemmA 4.3. Let $R$ be a G-semiprime ring, $H$ a subgroup of $G$ and $I$ a nonzero $H$-invariant ideal of $R$. Suppose that $I^{x} I=0$ for all $x \in G \backslash H$. Then:

(i) $G_{I}=H$;

(ii) $I$ is a trivial intersection ideal;

(iii) if $X$ is a right transversal for $H$ in $G$, then $I^{G}=\oplus \sum_{x \in X} I^{x}$.

Proof. Since $R$ is $G$-semiprime, Lemma 2.5(ii) implies that $I$ is $H$-nilpotent-free and hence that $I \cap l_{R}(I)=0$. In particular, $I^{2} \neq 0$ so (i) is immediate. Next observe that

$$
I \cap\left(\sum_{x \in G \backslash H} I^{x}\right) \subseteq I \cap l_{R}(I)=0
$$

and this clearly yields the direct sum in (iii). Since (ii) follows from (iii), the lemma is proved.

COROLlaRY 4.4. Let $R * G$ be a strongly $G$-graded ring whose base ring $R$ is $G$-semiprime. Then $R * G$ contains nonzero ideals $A, B$ with $A B=0$ if and only if there exist:

(i) a trivial intersection ideal I of $R$;

(ii) a finite group $N$ with normalizer $\mathbf{N}_{G}(N)=G_{l}$;

(iii) nonzero $G_{I}$-invariant ideals $\tilde{A}, \tilde{B}$ of $R * N$ with $\tilde{A}, \tilde{B} \subseteq I(R * N)$ and $\tilde{A} \tilde{B}=0$.

Furthermore, $A=B$ if and only if $\tilde{A}=\tilde{B}$. 
Proof. If $I, N, \tilde{A}$ and $\tilde{B}$ exist, then so do $A$ and $B$ by Theorem 1.3. Conversely suppose $A$ and $B$ exist. We apply Theorem 1.3 and use its notation. In particular, $N$ is a finite group and $N \subseteq H \subseteq \mathbf{N}_{G}(N)=\bar{H}$. Since $I^{x} I=0$ for all $x \in G \backslash \bar{H} \subseteq$ $G \backslash H$, Lemma 2.5 (i) implies that $\bar{I}=I^{\bar{H}}$ is an $\bar{H}$-invariant ideal of $R$ with $\bar{I}^{x} \bar{I}=0$ for all $x \in G \backslash \bar{H}$. By Lemma 4.3, $\bar{I}$ is a trivial intersection ideal with stabilizer $G_{\bar{I}}=\bar{H}=\mathbf{N}_{G}(N)$.

Now we consider the action of $\bar{H} \supseteq H$ on $R * N$. Set $\bar{A}=\tilde{A}^{\bar{H}}$ and $\bar{B}=\tilde{B}^{\bar{H}}$ so that these are $\bar{H}$-invariant ideals of $R * N$ clearly contained in $\bar{I}(R * N)$. Since $I^{x} I=0$ for all $x \in \bar{H} \backslash H$ and since $N \subseteq H$, it follows easily that $(I(R * N))^{x} \cdot I(R * N)=0$ for all $x \in \bar{H} \backslash H$ and hence that $\tilde{A}^{x} \tilde{B}=0$ for all such $x$. But $\tilde{A}$ is $H$-invariant and $\tilde{A} \tilde{B}=0$ so this yields $\tilde{A}^{\bar{H}} \tilde{B}=0$. Hence, since the right annihilator of $\tilde{A}^{\bar{H}}$ is $\bar{H}$ invariant, we conclude that $\bar{A} \bar{B}=\tilde{A}^{\bar{H}} \tilde{B}^{\bar{H}}=0$.

As is to be expected, the main theorem is used to obtain sufficient conditions for $R * G$ to be prime or semiprime. The real stumbling block to obtaining a precise answer in either case is that the answers for $G$ finite are just not satisfactory. This is so even if $R * G$ is assumed to be a crossed product. Thus in [8], it is shown that the primeness of a finite crossed product eventually depends on the $G$-primeness of certain suitably constructed twisted group algebras. But even the question of primeness of a finite twisted group algebra is unbelievably complicated. Indeed, the best result [7] requires the full classification of the finite simple groups. We finesse the problem by merely assuming that the finite groups which occur are all trivial.

Let $G$ be an arbitrary group. We denote by $\Delta^{+}(G)$ the join of all the finite normal subgroups of $G$. In particular, $\Delta^{+}(G)=\langle 1\rangle$ if and only if $G$ has no nontrivial finite normal subgroups.

COROLlaRY 4.5. Let $R * G$ be a strongly $G$-graded ring whose base ring $R$ is $G$-prime. Suppose that, for every trivial intersection ideal I of $R$, we have $\Delta^{+}\left(G_{I}\right)=\langle 1\rangle$. Then $R * G$ is prime.

Proof. We use Corollary 4.4. Suppose $I$ is a trivial intersection ideal of $R$ and $N$ is a finite subgroup of $G$ with $\mathbf{N}_{G}(N)=G_{I}$. Then $N \subseteq \Delta^{+}\left(G_{I}\right)=\langle 1\rangle$, by assumption, so clearly $N=\langle 1\rangle, R * N=R$ and $G_{I}=\mathbf{N}_{G}(N)=G$. Since $R$ is $G$-prime, it follows that $R * N=R$ can contain no nonzero $G$-invariant ideals $\tilde{A}$ and $\tilde{B}$ with $\tilde{A} \tilde{B}=0$. Thus by Corollary $4.4, R * G$ is prime.

Observe that if $G$ is torsion free, then the hypothesis $\Delta^{+}\left(G_{I}\right)=\langle 1\rangle$ is clearly satisfied. Thus we obtain

COROllaRY 4.6. Let $R * G$ be a strongly $G$-graded ring whose base ring $R$ is $G$-prime. If $G$ is torsion free, then $R * G$ is prime.

If $R * G$ is a crossed product, then this is Theorem II of [18]. In a different direction, suppose that $R$ is a prime ring so that it is certainly $G$-semiprime. Let $I$ be any trivial intersection ideal of $R$ with $G_{I}=H$. From $I^{x} I=0$ for all $x \in G \backslash H$, we conclude that $H=G$ and thus $I$ is merely a $G$-invariant ideal of $R$. It now follows that if (i), (ii) and (iii) of Corollary 4.4 are satisfied for $I$, then they are satisfied for 
$I=R$. Corollary 4.4 therefore yields

COROLlaRY 4.7. Let $R * G$ be a strongly G-graded ring whose base ring $R$ is prime. Then $R * G$ is prime (or semiprime) if and only if, for every finite normal subgroup $N$ of $G, R * N$ is $G$-prime (or $G$-semiprime, respectively).

When $R * G$ is a crossed product, this is Theorem 1.9 of [10].

5. Maschke's theorem. It remains to obtain an appropriate sufficient condition for $R * G$ to be semiprime. Again the precise answer eludes us because of finite group complications, but the results here are not too bad. Indeed for $R * G$ a finite crossed product over a semiprime ring, we need only assume that $R$ has no $|G|$-torsion to deduce that $R * G$ is semiprime [6]. Furthermore, the analogous result holds for strongly $G$-graded rings $[2,11]$. We actually require an operator version of this fact which does not readily follow from the known results. It does, however, follow easily from the techniques of [17].

We study (right) $R * G$-modules and we use $W \operatorname{ess}_{S} V$ to indicate that $W$ is essential as an $S$-submodule of $V$.

Lemma 5.1. Let $R * G$ be a strongly $G$-graded ring, let $V$ be an $R * G$-module and let $W$ be an $R$-submodule. Then:

(i) $W R_{x}$ is an $R$-submodule for all $x \in G$;

(ii) if $W \operatorname{ess}_{R} V$, then $W R_{x} \operatorname{ess}_{R} V$;

(iii) $\bigcap_{x \in G} W R_{x}$ is an $R * G$-submodule of $V$ contained in $W$.

Proof. (i) is clear since $R_{x} R=R_{x}$. For (ii) suppose that $U$ is a nonzero $R$-submodule of $V$. Since $R_{x^{-1}} R_{x}=R$, it follows from (i) that $U R_{x^{-1}}$ is also a nonzero $R$-submodule. Thus since $W \operatorname{ess}_{R} V$ we have $W \cap U R_{x^{-1}} \neq 0$ so

$$
W R_{x} \cap U \supseteq\left(W \cap U R_{x^{-1}}\right) R_{x} \neq 0 .
$$

Finally if $v \in \bigcap_{x \in G} W R_{x}$ and $y \in G$, then $v R_{y} \subseteq W R_{x} R_{y}=W R_{x y}$ for all $x \in G$ so

$$
v R_{y} \subseteq \bigcap_{x \in G} W R_{x y}=\bigcap_{x \in G} W R_{x}
$$

and the lemma is proved.

The next result is the version of Maschke's theorem which is known to hold in the context of strongly $G$-graded rings (see, for example, [11]). If $G$ is finite, an additive group $V$ is said to have no $|G|$-torsion if $v|G|=0$ for $v \in V$ implies that $v=0$. If $V$ is an $R * G$-module, we denote by $V_{R}$ the restriction of $V$ to $R$.

LEMMA 5.2. Let $R * G$ be a strongly $G$-graded ring with $G$ finite. Suppose that $W \subseteq V$ are $R * G$-modules with no $|G|$-torsion and that $W_{R}$ is a direct summand of $V_{R}$. Then there exists an $R * G$-submodule $U$ of $V$ with $(W \oplus U) \operatorname{ess}_{R} V$. Furthermore if $V=V \cdot|G|$, then $W$ is a direct summand of $V$.

Proof. For each $x \in G$ we have $1 \in R=R_{x} R_{x^{-1}}$ so we can write $1=\sum_{i} a_{x i} b_{x^{-1} i}$, a finite sum with $a_{x i} \in R_{x}$ and $b_{x^{-1} i} \in R_{x^{-1}}$. But assumption $W_{R}$ is a direct summand 
of $V_{R}$, so there exists an $R$-projection $\pi: V \rightarrow W$. We then define $\lambda: V \rightarrow W$ by

$$
v^{\lambda}=\sum_{x \in G} \sum_{i}\left(v a_{x i}\right)^{\pi} b_{x^{-1} i}
$$

for all $v \in V$. Since $W$ is an $R * G$-submodule, $\lambda$ is clearly an additive map from $V$ to $W$.

We claim that $\lambda$ is an $R * G$-homomorphism. To this end, let $\alpha_{y} \in R_{y}$ and consider $\left(v \alpha_{y}\right)^{\lambda}$. Observe that, for each $x \in G, \alpha_{y} a_{x i} \in R_{y x}$ and then that

$$
\alpha_{y} a_{x i}=\left(\sum_{j} a_{(y x) j} b_{(y x)^{-1} j}\right) \cdot\left(\alpha_{y} a_{x i}\right) \text {. }
$$

Furthermore, since $b_{(y x)^{-1} j}\left(\alpha_{y} a_{x i}\right) \in R, \pi$ is an $R$-homomorphism and $\sum_{i} a_{x i} b_{x^{-1} i}=1$, we have

$$
\begin{aligned}
\left(v \boldsymbol{\alpha}_{y}\right)^{\lambda} & =\sum_{x, i}\left(v \alpha_{y} a_{x i}\right)^{\pi} b_{x^{-1} i} \\
& =\sum_{x, i, j}\left(v a_{(y x) j} b_{(y, x)^{-1} j} \alpha_{y} a_{x i}\right)^{\pi} b_{x^{-1} i} \\
& =\sum_{x, i, j}\left(v a_{(y x) j}\right)^{\pi} b_{(y x)^{-1} j} \alpha_{y} \cdot a_{x i} b_{x^{-1} i} \\
& =\sum_{x, j}\left(v a_{(y x) j}\right)^{\pi} b_{(y, x)^{-1} j} \cdot \alpha_{y} \\
& =\left(\sum_{x, j}\left(v a_{x j}\right)^{\pi} b_{x^{-1} j}\right) \cdot \alpha_{y}=v^{\lambda} \cdot \alpha_{y} .
\end{aligned}
$$

Thus $\lambda$ is an $R * G$-homomorphism and hence if $U=\operatorname{ker} \lambda$ then $U$ is an $R * G$ submodule of $V$.

Set $n=|G|$ and observe that for $w \in W$ we have $w a_{x i} \in W$ so $\left(w a_{x i}\right)^{\pi}=w a_{x i}$ and therefore

$$
w^{\lambda}=\sum_{x, i} w a_{x i} b_{x^{-1} i}=\sum_{x} w=w n
$$

Thus since $V$ has no $n$-torsion we see that $W \cap U=0$. Now let $v \in V$ and set $w=v^{\lambda} \in W$. Then $(v n)^{\lambda}=v^{\lambda} n=w^{\lambda}$ so $(v n-w)^{\lambda}=0$ and we deduce that $V n \subseteq$ $W \oplus U$. In particular, if $X$ is an $R$-submodule of $V$ with $(W \oplus U) \cap X=0$, then $X n \subseteq(W \oplus U) \cap X=0$ and, since $V$ has no $n$-torsion, we conclude that $X=0$. Thus $(W \oplus U) \operatorname{ess}_{R} V$. Finally if $V=V n$, then $V=W \oplus U$.

As a consequence we obtain the essential version of Maschke's theorem.

Proposition 5.3. Let $R * G$ be a strongly $G$-graded ring with $G$ finite. Suppose that $W \subseteq V$ are $R * G$-modules with no $|G|$-torsion. Then $W \operatorname{ess}_{R * G} V$ if and only if $W \operatorname{ess}_{R} V$.

Proof. If $W \operatorname{ess}_{R} V$ then certainly $W \operatorname{ess}_{R * G} V$. Conversely, suppose $W \operatorname{ess}_{R *{ }_{G}} V$ and let $L$ be an $R$-submodule of $V$ maximal with respect to $W \cap L=0$. Then $(W \oplus L)$ ess $_{R} V$ and hence, by Lemma 5.1(ii), $(W \oplus L) R_{x} \operatorname{ess}_{R} V$ for all $x$. Moreover, 
if $E$ is the finite intersection $E=\bigcap_{x \in G}(W \oplus L) R_{x}$, then $E \operatorname{ess}_{R} V$ and $E$ is an $R * G$-module by Lemma 5.1(iii). Since $W$ is an $R * G$-submodule of $V$, we see that $W \subseteq E \subseteq W \oplus L$ and hence $E=W \oplus(E \cap L)$. In other words, $W_{R}$ is a direct summand of $E_{R}$. Thus Lemma 5.2 applies and there exists an $R * G$-submodule $U$ of $E$ with $(W \oplus U) \operatorname{ess}_{R} E$. Hence $(W \oplus U) \operatorname{ess}_{R} V$. But $W \operatorname{ess}_{R * G} V$ and $W \cap U=0$, so $U=0$ and the result follows.

It is now clear that much of the crossed product work of [17] carries over to strongly $G$-graded rings. However, this is not the appropriate place to pursue this line of thought. Rather we will content ourselves with obtaining the necessary operator version of the Fisher-Montgomery theorem. It is clear that the following proof only requires that $H$ strongly permutes the ideals of $R * G$ and of $R$ in a suitably compatible manner. However we will simply state the result in the form we will use it.

LEMMA 5.4. Let $R * H$ be a strongly $H$-graded ring, let $G$ be a finite normal subgroup of $H$ and let $I$ be an $H$-invariant ideal of $R$. If $I$ is $H$-nilpotent-free with no $|G|$-torsion, then $I(R * G)$ is an $H$-nilpotent-free ideal of $R * G$.

Proof. We first observe that $I(R * G)$ has no $|G|$-torsion. Indeed suppose $\gamma$ $=\sum_{x} \gamma_{x} \in I(R * G)$ with $|G| \gamma=0$. Then, for all $x \in G$ we have $|G| \gamma_{x} R_{x^{-1}}=0$ and $\gamma_{x} R_{x^{-1}} \subseteq I$. Since $I$ has no $|G|$-torsion, we conclude that $\gamma_{x} R_{x^{-1}}=0$ and hence that $\gamma=0$.

Now suppose $A$ is an $H$-invariant ideal of $R * G$ contained in $I(R * G)$ with $A^{2}=0$. If $L=l_{I(R * G)}(A)$, then $L$ is a two-sided ideal of $R * G$. Furthermore, as right $(R * G)$-modules, we have $L \operatorname{ess}_{R * G} I(R * G)$. Indeed suppose $K$ is a nonzero right ideal of $R * G$ contained in $I(R * G)$. If $K A \neq 0$, then $A^{2}=0$ implies that $0 \neq K A \subseteq L \cap K$. On the other hand, if $K A=0$ then $0 \neq K \subseteq L \cap K$.

Since $L \operatorname{ess}_{R * G} I(R * G)$ and $I(R * G)$ has no $|G|$-torsion, we deduce from Proposition 5.3 that $L \operatorname{ess}_{R} I(R * G)$ and therefore that $L \cap I=l_{I}(A)$ is essential, as a right $R$-module, in $I \subseteq I(R * G)$. Now observe that $L^{\prime}=l_{I}(A)$ is an $H$-invariant ideal of $R$ contained in $I$ and that $I$ is $H$-nilpotent-free, by assumption. Thus $L^{\prime} \cap \underline{r}_{I}\left(L^{\prime}\right)=0$ and since $L^{\prime} \operatorname{ess}_{R} I$ we have $\underline{r}_{I}\left(L^{\prime}\right)=0$. Finally, if $\alpha=\sum \alpha_{x} \in A \subseteq I(R * G)$, then $L^{\prime} \alpha=0$ implies that $L^{\prime} \alpha_{x} R_{x^{-1}}=0$ for all $x$. Thus since $\alpha_{x} R_{x^{-1}} \subseteq I$ we have $\alpha_{x} R_{x^{-1}} \subseteq \underline{r}_{I}\left(L^{\prime}\right)=0$ and therefore $\alpha=0$. We conclude that $A=0$ and hence that $I(R * G)$ is $H$-nilpotent-free.

If $V$ is an additive abelian group and $G$ is arbitrary, then we say that $V$ has no $|G|$-torsion if, for all finite subgroups $N$ of $G, V$ has no $|N|$-torsion. We can now quickly prove

COROLlaRY 5.5. Let $R * G$ be a strongly $G$-graded ring whose base ring $R$ is $G$-semiprime. Suppose that, for every trivial intersection ideal $I$ of $R, I$ has no $\left|\Delta^{+}\left(G_{I}\right)\right|$-torsion. Then $R * G$ is semiprime.

Proof. Let $I$ be any trivial intersection ideal of $R$ and set $H=G_{I}$. Then $I^{x} I \subseteq I^{x} \cap I=0$ for all $x \in G \backslash H$ so Lemma 2.5 (ii) implies that $I$ is $H$-nilpotentfree. Suppose $N$ is a finite normal subgroup of $H$. Then $N \subseteq \Delta^{+}\left(G_{I}\right)$ so, by 
assumption, $I$ has no $|N|$-torsion. We can therefore conclude from Lemma 5.4 that $I(R * N)$ is an $H$-nilpotent-free ideal of $R * N$ and Corollary 4.4 yields the result.

Observe that if $R$ has no $|G|$-torsion, then certainly $I$ has no $\left|\Delta^{+}\left(G_{I}\right)\right|$-torsion for any ideal $I$ of $R$. Thus we obtain

COROLlary 5.6. Let $R * G$ be a strongly G-graded ring whose base ring $R$ is $G$-semiprime. If $R$ has no $|G|$-torsion, then $R * G$ is semiprime.

If $R * G$ is a crossed product, then this is Theorem I of [18]. Furthermore, since any semiprime ring is $G$-semiprime, this includes the main result of [16].

Finally, we remark that the results of this paper have been extended to more general group-graded rings by D. Quinn [19] using a refinement of the duality developed in [2]. In addition, that paper contains a much less computational proof of the essential version of Maschke's theorem.

\section{REFERENCES}

1. G. Azumaya, New foundations of the theory of simple rings, Proc. Japan Acad. 22 (1946), 325-332.

2. M. Cohen and S. Montgomery, Group-graded rings, smash products, and group actions, Trans. Amer. Math. Soc. 273 (1982), 131-145.

3. M. Cohen and L. Rowen, Group-graded rings, Comm. Algebra 11 (1983), 1253-1270.

4. I. G. Connell, On the group ring, Canad. J. Math. 15 (1963), 650-685.

5. E. C. Dade, Group graded rings and modules, Math. Z. 174 (1980), 241-262.

6. J. W. Fisher and S. Montgomery, Semi-prime skew group rings, J. Algebra 52 (1978), 241-247.

7. R. B. Howlett and I. M. Isaacs, On groups of central type, Math. Z. 179 (1982), 555-569.

8. M. Lorenz and D. S. Passman, Prime ideals in crossed products of finite groups, Israel J. Math. 33 (1979), 89-132; 35 (1980), 311-322.

9. S. Montgomery, Outer automorphisms of semiprime rings, J. London Math. Soc. (2) 18 (1978), 209-221.

10. S. Montgomery and D. S. Passman, Crossed products over prime rings, Israel J. Math. 31 (1978), 224-256.

11. C. Nastacescu, Strongly graded rings of finite groups, Comm. Algebra 11 (1983), 1033-1071.

12. C. Nastacescu and F. Van Oystaeyen, On strongly graded rings and crossed products, Comm. Algebra 10 (1982), 2085-2106.

13. D. S. Passman, Nil ideals in group rings, Michigan J. Math. 9 (1962), 375-384.

14. _ Radicals of twisted group rings, Proc. London Math. Soc. 20 (1970), 409-437.

15. The algebraic structure of group rings, Interscience, New York, 1977.

16. __ Crossed products over semiprime rings, Houston Math. J. 4 (1978), 583-592.

17. I_ It's essentially Maschke's theorem, Rocky Mountain J. Math. 13 (1983), 37-54.

18. Semiprime and prime crossed products, J. Algebra 83 (1983), 158-178.

19. D. Quinn, Group-graded rings and duality (to appear).

Department of Mathematics, University of Wisconsin, Madison, Wisconsin 53706 